\title{
6.3.A Sensitivity Data File Formats
}

\subsection{A.1 Format of TSUNAMI-A sensitivity data file}

The format of the TSUNAMI-A sensitivity data file produced by SAMS for cases with deterministic transport solutions is given in Table 6.3.A.1. The occurrence of each entry in the data file is followed by an identification of the data contained on each line of the file and the FORTRAN edit descriptor denoting the format of each line. A brief description of each line is also presented.

A sample of the TSUNAMI-A data file for the Flattop-25 sample problem is provided in Figure 6.3.A.1. Here, only two profiles out of the 130 computed are shown. 
Table 6.3.A.1. Format specification for TSUNAMI-A sensitivity data file

\begin{tabular}{|c|c|c|c|}
\hline Occurrence & Data & Format & Description \\
\hline \multirow{6}{*}{$\begin{array}{l}\text { Once at beginning } \\
\text { of file. }\end{array}$} & title & $\mathrm{a} 80$ & $\begin{array}{l}\text { Title extracted from transport } \\
\text { calculation }\end{array}$ \\
\hline & number of neutron groups & i10 & $\begin{array}{l}\text { Number of neutron groups in } \\
\text { calculation }\end{array}$ \\
\hline & $\begin{array}{l}\text { total number of profiles, } \\
\text { text descriptor, } \\
\text { number of total profiles that are } \\
\text { region integrated }\end{array}$ & i10, a35, i10 & $\begin{array}{l}\text { Total number of sensitivity } \\
\text { profiles in data file, separated by a } \\
\text { text descriptor, then the number of } \\
\text { profiles that contain region- } \\
\text { integrated data }\end{array}$ \\
\hline & $k_{\text {eff }}$ & $\mathrm{f} 10.6$ & $\begin{array}{l}\text { Value of } k_{\text {eff }} \text { from the forward } \\
\text { transport calculation }\end{array}$ \\
\hline & 'energy boundaries:' & $\mathrm{a}$ & Text \\
\hline & energy boundary data & $\begin{array}{l}\text { 5es14.6 } \\
\text { (repeats until } \\
\text { all data is } \\
\text { printed) }\end{array}$ & $\begin{array}{l}\text { Values of boundaries of energy } \\
\text { groups. Begins with upper value } \\
\text { for highest energy group and ends } \\
\text { with lower value of lowest energy } \\
\text { group. }\end{array}$ \\
\hline \multirow{3}{*}{$\begin{array}{l}\text { Repeats for each } \\
\text { profile. }\end{array}$} & $\begin{array}{l}\text { isotope name, } \\
\text { sensitivity reaction name, } \\
\text { nuclide ID, } \\
\text { MT number, } \\
\text { Zone number or negative of material } \\
\text { number, } \\
\text { zone volume }\end{array}$ & $\begin{array}{l}\text { a12, 1x, a15, } \\
3 i 12, \text { es14.6 }\end{array}$ & $\begin{array}{l}\text { Provides data identifying the } \\
\text { sensitivity data that follows. } \\
\text { Note, if sensitivity data is region- } \\
\text { integrated, zone number and zone } \\
\text { volume are both } 0 \text {. If data is } \\
\text { integrated over all zones } \\
\text { containing the same material, the } \\
\text { material number is given in place } \\
\text { of the zone number as a negative } \\
\text { number. }\end{array}$ \\
\hline & $\begin{array}{l}\text { Energy integrated sensitivity } \\
\text { coefficient, sum of absolute value of } \\
\text { group-wise sensitivities, sum of the } \\
\text { group-wise sensitivities with } \\
\text { opposite sign as energy integrated } \\
\text { value (osc) }\end{array}$ & 3es14.6 & $\begin{array}{l}\text { Energy-integrated sensitivity } \\
\text { coefficients for this profile. }\end{array}$ \\
\hline & group-wise sensitivity coefficients & $\begin{array}{l}\text { 5es14.6 } \\
\text { (repeats until } \\
\text { all data is } \\
\text { printed) }\end{array}$ & $\begin{array}{l}\text { Energy-dependent sensitivity } \\
\text { coefficients. Begins with highest } \\
\text { energy group. }\end{array}$ \\
\hline Once at end of file. & & & $\begin{array}{l}\text { Block of file verification } \\
\text { information. }\end{array}$ \\
\hline
\end{tabular}


flat top- 25

238 number of neutron groups

130 number of sensitivity profiles

1.002969 $k$-eff from the forward case
30 are region integrated energy boundaries:

2. $000000 \mathrm{E}+07$

1. $284000 \mathrm{E}+07$

4. $304000 \mathrm{E}+06$

$1.500000 \mathrm{E}+06$

$1.200000 \mathrm{E}+06$

$8.750000 \mathrm{E}+05$

$6.700000 \mathrm{E}+05$

$4.700000 \mathrm{E}+05$

$2.700000 \mathrm{E}+05$

$8.500000 \mathrm{E}+04$

$5.200000 \mathrm{E}+04$

1. $7000000 \mathrm{E}+04$

$3.900000 \mathrm{E}+03$

$2.200000 \mathrm{E}+03$

$9.500000 \mathrm{E}+02$

$2.850000 \mathrm{E}+02$

1. $860000 \mathrm{E}+02$

1. $0000000 \mathrm{E}+\odot 2$

7. $200000 \mathrm{E}+01$

$5.340000 \mathrm{E}+01$

4. $700000 \mathrm{E}+01$

$3.960000 \mathrm{E}+01$

3. $460000 \mathrm{E}+01$

3. $0000000 \mathrm{E}+01$

2. $000000 \mathrm{E}+01$

1. $509990 \mathrm{E}+01$

$1.150000 \mathrm{E}+01$

7. $.000000 \mathrm{E}+00$

$5.400000 \mathrm{E}+00$

3. $500000 \mathrm{E}+00$

$2.870000 \mathrm{E}+00$

$2.379990 \mathrm{E}+0 \odot$

$1.940000 \mathrm{E}+00$

$1.500000 \mathrm{E}+00$

$1.250000 \mathrm{E}+0 \odot$

$1.139990 \mathrm{E}+00$

$1.089990 \mathrm{E}+0 \odot$

1. $040000 \mathrm{E}+00$

9. $750000 \mathrm{E}-01$

8. $0000000 \mathrm{E}-01$

6. $0000000 \mathrm{E}-01$

3. $750000 \mathrm{E}-01$

2. $5000000 \mathrm{E}-01$
1. $733300 \mathrm{E}+07$

1. $0000000 \mathrm{E}+07$

$3.000000 \mathrm{E}+06$

1. $400000 \mathrm{E}+\odot 6$

$1.100000 \mathrm{E}+06$

8. $611000 \mathrm{E}+05$

6. $000000 \mathrm{E}+05$

$4.400000 \mathrm{E}+05$

2. $000000 \mathrm{E}+05$

8. $200000 \mathrm{E}+04$

$5.000000 \mathrm{E}+04$

1. $3000000 \mathrm{E}+04$

$3.740000 \mathrm{E}+03$

$1.800000 \mathrm{E}+03$

$6.830000 \mathrm{E}+02$

$2.400000 \mathrm{E}+02$

1. $220000 \mathrm{E}+02$

9. $000000 \mathrm{E}+01$

6. $750000 \mathrm{E}+01$

5. $200000 \mathrm{E}+01$

4. $520000 \mathrm{E}+01$

3. $910000 \mathrm{E}+01$

3. $375000 \mathrm{E}+01$

2. $750000 \mathrm{E}+01$

1. $900000 \mathrm{E}+01$

1. $440000 \mathrm{E}+01$

1. $000000 \mathrm{E}+01$

$6.750000 \mathrm{E}+00$

$5.000000 \mathrm{E}+0 \odot$

$3.150000 \mathrm{E}+00$

$2.770000 \mathrm{E}+00$

$2.299990 \mathrm{E}+00$

$1.860000 \mathrm{E}+\odot \odot$

1. $450000 \mathrm{E}+0 \odot$

1. $224990 \mathrm{E}+\odot \odot$

$1.129990 \mathrm{E}+\odot \odot$

$1.080000 \mathrm{E}+00$

$1.030000 \mathrm{E}+00$

9. $500000 \mathrm{E}-01$

7. 500000อ - 01

5. 500000E- 01

3. 500000E- 01

2. 25000०E-01
$1.568300 \mathrm{E}+07$

$8.187300 \mathrm{E}+06$

$2.479000 \mathrm{E}+06$

$1.356000 \mathrm{E}+06$

1. $010000 \mathrm{E}+06$

$8.200000 \mathrm{E}+05$

$5.730000 \mathrm{E}+05$

4. $200000 \mathrm{E}+05$

$1.500000 \mathrm{E}+05$

$7.500000 \mathrm{E}+04$

$4.500000 \mathrm{E}+04$

$9.5000000 \mathrm{E}+03$

$3.0000000 \mathrm{E}+03$

$1.550000 \mathrm{E}+03$

$6.700000 \mathrm{E}+02$

$2.100000 \mathrm{E}+02$

1. $190000 \mathrm{E}+02$

8. $2000000 \mathrm{E}+01$

$6.500000 \mathrm{E}+01$

5. $0600000 \mathrm{E}+01$

$4.4000000 \mathrm{E}+01$

$3.800000 \mathrm{E}+01$

$3.325000 \mathrm{E}+01$

$2.500000 \mathrm{E}+01$

$1.850000 \mathrm{E}+01$

$1.375000 \mathrm{E}+01$

$9.099990 \mathrm{E}+\odot \odot$

$6.500000 \mathrm{E}+00$

$4.750000 \mathrm{E}+00$

3. $\odot 49990 \mathrm{E}+\odot \odot$

2. $669990 \mathrm{E}+00$

2. $209990 \mathrm{E}+0 \odot$

$1.770000 \mathrm{E}+00$

$1.400000 \mathrm{E}+00$

$1.200000 \mathrm{E}+00$

$1.120000 \mathrm{E}+00$

$1.070000 \mathrm{E}+0 \odot$

1. $020000 \mathrm{E}+00$

9. $250000 \mathrm{E}-01$

7. $0000000 \mathrm{E}-01$

5. $0000000 \mathrm{E}-01$

3. 250000E- 01

2. $0000000 \mathrm{E}-01$
1. $455000 \mathrm{E}+07$

$6.434000 \mathrm{E}+06$

$2.354000 \mathrm{E}+06$

1. $317000 \mathrm{E}+06$

$9.200000 \mathrm{E}+05$

$7.500000 \mathrm{E}+05$

$5.500000 \mathrm{E}+05$

$4.000000 \mathrm{E}+05$

1. $283000 \mathrm{E}+05$

7. $300000 \mathrm{E}+04$

3. $0000000 \mathrm{E}+04$

$8.030000 \mathrm{E}+03$

$2.580000 \mathrm{E}+03$

$1.500000 \mathrm{E}+03$

$5.500000 \mathrm{E}+02$

2. $075000 \mathrm{E}+02$

1. $150000 \mathrm{E}+02$

$8.000000 \mathrm{E}+01$

$6.100000 \mathrm{E}+01$

$4.920000 \mathrm{E}+01$

4. $240000 \mathrm{E}+01$

3. $700000 \mathrm{E}+01$

$3.175000 \mathrm{E}+01$

2. $250000 \mathrm{E}+01$

$1.700000 \mathrm{E}+01$

1. $290000 \mathrm{E}+01$

$8.099990 \mathrm{E}+\odot \odot$

$6.250000 \mathrm{E}+\odot \odot$

4. $0000000 \mathrm{E}+\odot \odot$

$3.000000 \mathrm{E}+00$

$2.570000 \mathrm{E}+00$

$2.120000 \mathrm{E}+00$

$1.679990 \mathrm{E}+\odot \odot$

1. $349990 \mathrm{E}+\odot \odot$

$1.174990 \mathrm{E}+\odot \odot$

$1.110000 \mathrm{E}+00$

$1.059990 \mathrm{E}+0 \odot$

1. $009990 \mathrm{E}+0 \odot$

$9.000000 \mathrm{E}-01$

$6.500000 \mathrm{E}-01$

4. 500000อE- 01

3. $000000 \mathrm{E}-01$

1. 750000E- 01
1. $3840000 \mathrm{E}+07$

4. $800000 \mathrm{E}+06$

$1.850000 \mathrm{E}+06$

1. $250000 \mathrm{E}+06$

$9.000000 \mathrm{E}+05$

$6.790000 \mathrm{E}+05$

$4.995200 \mathrm{E}+05$

$3.300000 \mathrm{E}+05$

1. $0000000 \mathrm{E}+05$

$6.000000 \mathrm{E}+04$

2. $500000 \mathrm{E}+04$

$6.000000 \mathrm{E}+03$

2. $290000 \mathrm{E}+03$

1. $150000 \mathrm{E}+03$

3. $050000 \mathrm{E}+02$

1. $925000 \mathrm{E}+02$

1. $080000 \mathrm{E}+02$

7. $600000 \mathrm{E}+01$

5. $900000 \mathrm{E}+01$

4. $830000 \mathrm{E}+01$

$4.100000 \mathrm{E}+01$

3. $550000 \mathrm{E}+01$

$3.125000 \mathrm{E}+01$

2. $100000 \mathrm{E}+01$

$1.600000 \mathrm{E}+01$

$1.190000 \mathrm{E}+01$

$7.150000 \mathrm{E}+00$

$6.000000 \mathrm{E}+00$

$3.730000 \mathrm{E}+0 \odot$

$2.969990 \mathrm{E}+0 \odot$

2. $469990 \mathrm{E}+0 \odot$

$2.000000 \mathrm{E}+00$

$1.589990 \mathrm{E}+\odot \odot$

1. $299990 \mathrm{E}+0 \odot$

$1.150000 \mathrm{E}+00$

1. $099990 \mathrm{E}+\odot \odot$

1. $049990 \mathrm{E}+00$

1. $000000 \mathrm{E}+00$

8. $500000 \mathrm{E}-01$

$6.250000 \mathrm{E}-01$

4. $000000 \mathrm{E}-01$

2.750000E- 01

$1.500000 \mathrm{E}-01$

Figure 6.3.A.1. Truncated sensitivity data file for Flattop- 25 sample problem. 


\begin{tabular}{|c|c|c|c|c|}
\hline $000 \mathrm{E}-01$ & $\odot \mathrm{E}-\odot 1$ & ๑०९००E - ०2 & 8. $.00000 \mathrm{E}-\odot 2$ & 7. $0000 \odot \odot E-\odot 2$ \\
\hline 6. $. \odot \odot \odot \odot \odot E-\odot 2$ & $5.0 \odot \odot \odot \odot \odot E-\odot 2$ & 4. $.0 \odot \odot \odot \odot \odot E-\odot 2$ & 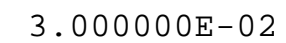 & $2.5300 \odot \odot E-\odot 2$ \\
\hline 1. $000 \odot \odot \odot E-\odot 2$ & $5 ค ค ค ค ค F$ & คคคคคค & $\triangle 1 ค ค ค ค ค ค ค$ & \\
\hline$\odot \odot E-\odot 3$ & 03 & $\odot \odot \odot \odot E$ & $1.2000 \odot \odot \mathrm{E}$ & 1.0 \\
\hline $7.500000 \mathrm{E}-\odot 4$ & 5. $\odot \odot \odot \odot \odot \odot E-\odot 4$ & $\odot \odot E-\odot 4$ & 1.06 & \\
\hline tota & & & 1 & $\odot$ \\
\hline $4.717915 \mathrm{E}-03$ & $6 E-03$ & $3 E-\odot 4$ & & \\
\hline $2 E-\odot 8$ & & & $3.212515 \mathrm{E}-07$ & 8.004 \\
\hline $9 \mathrm{E}-\odot 6$ & $2 E-05$ & $3 E-\odot 5$ & $5243 E-04$ & $235-81$ \\
\hline $243 E-04$ & $4.044642 E-\odot 4$ & $1 E-\odot 4$ & $5.606487 \mathrm{E}-\odot 4$ & 4.75 \\
\hline $1 E-\odot 4$ & $6.761932 \mathrm{E}-05$ & $3 E-05$ & 1. $095966 \mathrm{E}-\odot 4$ & $8.561461 \mathrm{E}-\odot 5$ \\
\hline $1.773753 \mathrm{E}-04$ & $1.640000 \mathrm{E}-\odot 4$ & $1.761175 \mathrm{E}-04$ & $4.353814 \mathrm{E}-05$ & $5.616768 \mathrm{E}-05$ \\
\hline $9 E-\odot 5$ & $6 E-\odot 4$ & $8 E-\odot 4$ & $96 E-\odot 4$ & $4 E-05$ \\
\hline 4 & $5 . \varepsilon$ & 5 & $2 E-04$ & \\
\hline & & & & \\
\hline 1.4 & -3.8 & -8.1 & $720 E-\odot 5$ & $4 E-05$ \\
\hline-1.8 & & $7 E-06$ & ๑5E- - 5 & $7 E-06$ \\
\hline$\odot E-\odot 6$ & $1 E-06$ & $\odot E-\odot 5$ & $57 E-06$ & $99 E-06$ \\
\hline$=-06$ & $-\odot 6$ & $E-\odot 6$ & $=-06$ & E- -6 \\
\hline 8 & -3 . & $E-\odot 7$ & $-\odot 7$ & $E-\odot 8$ \\
\hline & & & & \\
\hline-3. & -1 . & -9.17 & -1.9 & \\
\hline & -2.7 & -7. & 3.1 & -2 . \\
\hline & $2 \mathrm{E}-12$ & $2 E-11$ & $1 E-10$ & $2 E-10$ \\
\hline-8 & -8.1 & -1.2 & -2 & 12 \\
\hline 2. & $3 . \varepsilon$ & 3.2 & -1.8 & 12 \\
\hline & -2 . & -1 . & -7 . & \\
\hline-2 & -2.4 & 4.30 & 1.32 & -2 . \\
\hline-3.5 & $-1 . c$ & 13 & 2. & -6 . \\
\hline & & & $E-11$ & \\
\hline 3.5 & -1 . & -6 . & 4. & 13 \\
\hline 7.6 & -3 & -1 & -1. & 14 \\
\hline & & & 1. & 13 \\
\hline$E-13$ & $88 \mathrm{E}-14$ & $97 \mathrm{E}-13$ & $-1.360891 E-13$ & $89 E-15$ \\
\hline 1.7 & -2 . & -1 & $\mathrm{E}-14$ & $4 \mathrm{E}-12$ \\
\hline & & & -1.2 & $\mathrm{LE}-13$ \\
\hline & & -5 & -5 & -2 \\
\hline & -2. & -2.4 & -2.2 & -1 . \\
\hline & -1. & $-1.2 \cdot \operatorname{se}(x)$ & & \\
\hline-7. & $17 E-14$ & $473 E-14$ & $883 E-15$ & $-9.776219 E-15$ \\
\hline & & -3.2 & 15 & $5 E-15$ \\
\hline & & & $1 E-17$ & $6 E-16$ \\
\hline-3.8 & -2.5 & -1 & -1.0 & $E-18$ \\
\hline & & -2.3 & -2.6 & \\
\hline & & & & -3 \\
\hline-6 . & -7. & $30 E-16$ & $348 E-15$ & $912 \mathrm{E}-15$ \\
\hline-1 & -1 & $-1.2 \cdot r y$ & $-2 . \varsigma$ & OE - 16 \\
\hline 2.1 & $21 E-15$ & $77 E-15$ & OE - 15 & $233 E-16$ \\
\hline 1.5 & 4.7 & & -1.3 & -7.3 \\
\hline $41 E-19$ & & & $7866 E-22$ & $6.410866 \mathrm{E}-23$ \\
\hline
\end{tabular}

Figure 6.3.A.1. Truncated sensitivity data file for Flattop-25 sample problem. (continued) 


\begin{tabular}{|c|c|c|c|c|}
\hline 24 & 5 & 6 & 26 & -5 \\
\hline $415888 F-27$ & $-3.110222 E-28$ & $-5.522761 E-29$ & $-5.429459 \mathrm{E}-30$ & $-1728100 \mathrm{~F}-30$ \\
\hline & $\odot . \odot \odot \odot \odot \odot \odot E+\odot \odot$ & $\odot . \odot \odot \odot \odot \odot \odot E+\odot \odot$ & & \\
\hline$\odot . \odot \odot \odot \odot \odot \odot E+\odot \odot$ & $\odot . \odot \odot \odot \odot \odot \odot E+\odot \odot$ & $\odot \odot \odot \odot \odot \odot E+\odot \odot$ & $\odot . \odot \odot \odot \odot \odot \odot E+\odot \odot$ & 0.0 \\
\hline$\odot . \odot \odot \odot \odot \odot \odot E+\odot \odot$ & $\odot . \odot \odot \odot \odot \odot \odot E+\odot \odot$ & $\odot \odot \odot E+\odot \odot$ & & \\
\hline 234 & & & 1 & 0 \\
\hline $8.032 \varepsilon$ & $8.241044 \mathrm{E}-\odot 4$ & 10๑6E- $\odot 5$ & & \\
\hline & & & 4.89458 & 8.37 \\
\hline 3. & 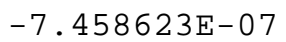 & -7. & & \\
\hline-05 & $3.092353 E-05$ & $9.673315 \mathrm{E}-\odot 6$ & ๑E - $\odot 5$ & 4.96 \\
\hline $5 E-05$ & $184 E-05$ & 1.13 & 83885E- 05 & $96 E-05$ \\
\hline & $4228 E-\odot 5$ & $3.382708 \mathrm{E}-05$ & $2870 E-\odot 6$ & 8. 988859E-०6 \\
\hline$=-06$ & 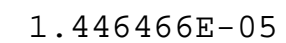 & 2.42 & & 06 \\
\hline & 1.3 & 1.1 & & 1. \\
\hline & & & & \\
\hline 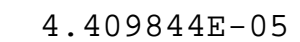 & $2 . \varepsilon$ & 1.0 & & \\
\hline & 1.7 & $E-\odot 7$ & 06 & $8 E-07$ \\
\hline & & $\mathrm{E}-\odot 7$ & $4 E-07$ & $6 E-07$ \\
\hline-2 . & -1 & -2.3 & -3. & $E-\odot 8$ \\
\hline-3. & -2.7 r & 1. & -1 . & 1. \\
\hline & -1.7 & 2.2 & & -5 . \\
\hline & -1 & -3.2 & & \\
\hline & -5 & $E-11$ & & 10 \\
\hline & & -1 & & \\
\hline 1. & $-4 . \varepsilon-\gamma-\gamma$ & $-9.2+x+2$ & 12 & -13 \\
\hline-1 & $3 . \varepsilon$ & $2 . \varepsilon$ & -1 . & \\
\hline-2 . & -1.7 r & -4.7 & & 4 \\
\hline & -6.3 & & 12 & -15 \\
\hline 5. & -7.8 & 2. & $E-12$ & $4 \mathrm{E}-15$ \\
\hline & & & & \\
\hline & -8. & 5. & & -13 \\
\hline$-4.8+2 x-3$. & -2.3 & -7.8 & -12 & $E-14$ \\
\hline-5 . & & 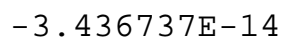 & & $E-13$ \\
\hline & 5.5 & -3. & & $E-15$ \\
\hline 13 & -1 & -3.3 & -14 & $1 E-13$ \\
\hline & & & & -8 . \\
\hline-14 & & 3. & -4. & -4 \\
\hline-1 . & -2. & -1.1 & -16 & -16 \\
\hline & -8.4 & -2.4 & $=-14$ & $4 E-15$ \\
\hline-16 & E-16 & $8 E-16$ & $=-16$ & $5 E-17$ \\
\hline-17 & $E-18$ & $1 E-16$ & $E-15$ & $3 E-15$ \\
\hline & & & 16 & $E-16$ \\
\hline & -9.86 & $-7.532523 E-18$ & $E-18$ & $4 E-18$ \\
\hline & -4.3 & & -6.6 & -6 . \\
\hline & & & & \\
\hline 6 & $528 F-18$ & $15 \mathrm{E}-17$ & $\mathrm{E}-17$ & $8 E-18$ \\
\hline $5 E-18$ & $E-18$ & $21 \mathrm{E}-17$ & $7 E-18$ & $-1.094495 E-18$ \\
\hline & -2.3 & $7 E-16$ & $E-16$ & $78 E-17$ \\
\hline E-18 & $1 E-18$ & $1.795543 \mathrm{E}-19$ & $35-19$ & $-2.070213 E-1$ \\
\hline
\end{tabular}

Figure 6.3.A.1. Truncated sensitivity data file for Flattop- 25 sample problem. (continued) 


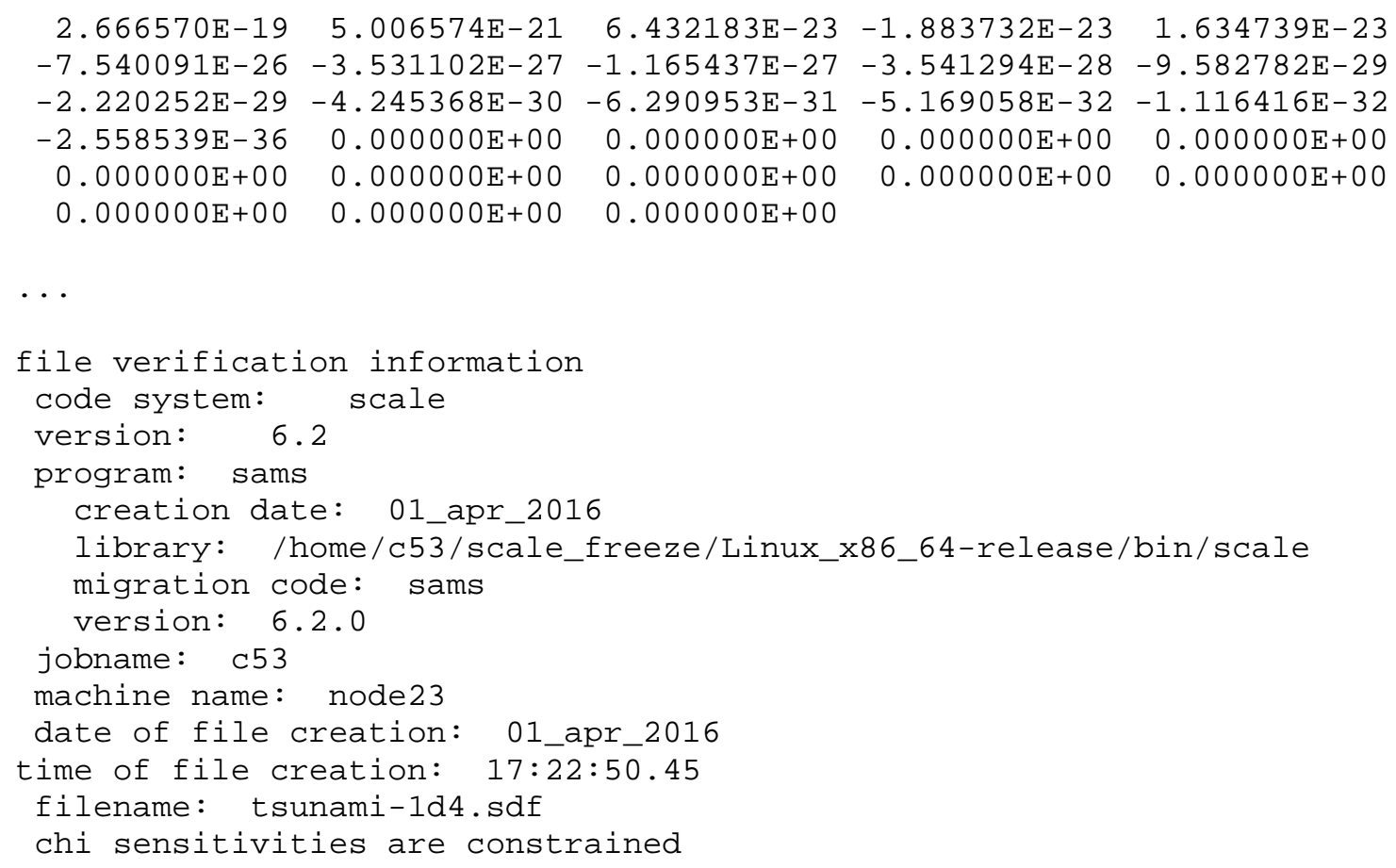

Figure 6.3.A.1. Truncated sensitivity data file for Flattop-25 sample problem. (continued)

\subsection{A.2 Format of TSUNAMI-B sensitivity data file}

The format of the TSUNAMI-B sensitivity data file produced by SAMS for cases with Monte Carlo transport solutions is given in Table 6.3.A.2. The occurrence of each entry in the data file is followed by an identification of the data contained on each line of the file and the FORTRAN edit descriptor denoting the format of each line. A brief description of each line is also presented.

A sample of the TSUNAMI-B data file for the LEU-COMP-THERM-009 case 10 sample problem is provided in Figure 6.3.A.2. Here, only two profiles out of the 3389 computed are shown. 
Table 6.3.A.2. Format specification for TSUNAMI-B sensitivity data file

\begin{tabular}{|c|c|c|c|}
\hline Occurrence & Data & Format & Description \\
\hline \multirow{6}{*}{$\begin{array}{l}\text { Once at beginning of } \\
\text { file. }\end{array}$} & title & $\mathrm{a} 80$ & $\begin{array}{l}\text { Title extracted from transport } \\
\text { calculation }\end{array}$ \\
\hline & number of neutron groups & i10 & $\begin{array}{l}\text { Number of neutron groups in } \\
\text { calculation }\end{array}$ \\
\hline & $\begin{array}{l}\text { total number of profiles, } \\
\text { text descriptor, } \\
\text { number of total profiles that are region } \\
\text { integrated }\end{array}$ & $\mathrm{i} 10, \mathrm{a} 35, \mathrm{i} 10$ & $\begin{array}{l}\text { Total number of sensitivity } \\
\text { profiles in data file, separated } \\
\text { by a text descriptor, then the } \\
\text { number of profiles that contain } \\
\text { region-integrated data }\end{array}$ \\
\hline & $k_{\text {eff. }}{ }^{\prime}+/$-' $^{\prime} \sigma$ & $\begin{array}{l}\mathrm{f} 10.6,1 \mathrm{x}, \mathrm{a}, \\
1 \mathrm{x}, \mathrm{f} 10.6\end{array}$ & $\begin{array}{l}\text { Value of } k_{\text {eff }} \text { from the forward } \\
\text { transport calculation and its } \\
\text { standard deviation }\end{array}$ \\
\hline & 'energy boundaries:' & $\mathrm{a}$ & Text \\
\hline & energy boundary data & $\begin{array}{l}\text { 5es14.6 (repeats } \\
\text { until all data is } \\
\text { printed) }\end{array}$ & $\begin{array}{l}\text { Values of boundaries of } \\
\text { energy groups. Begins with } \\
\text { upper value for highest energy } \\
\text { group and ends with lower } \\
\text { value of lowest energy group. }\end{array}$ \\
\hline \multirow{3}{*}{$\begin{array}{l}\text { Repeats for each } \\
\text { profile. }\end{array}$} & $\begin{array}{l}\text { isotope name, } \\
\text { sensitivity reaction name, } \\
\text { nuclide ID, } \\
\text { MT number }\end{array}$ & $\begin{array}{l}\text { a12, 1x, a15, } \\
\text { 2i12 }\end{array}$ & $\begin{array}{l}\text { Provides data identifying the } \\
\text { sensitivity data that follows. } \\
\text { Note, if sensitivity data is } \\
\text { region-integrated, zone } \\
\text { number and zone volume are } \\
\text { both 0. If data is integrated } \\
\text { over all zones containing the } \\
\text { same material, the material } \\
\text { number is given in place of the } \\
\text { zone number as a negative } \\
\text { number. }\end{array}$ \\
\hline & $\begin{array}{l}\text { Unit number, } \\
\text { number of the region within the unit, } \\
\text { unit comments }\end{array}$ & $2 \mathrm{i} 7, \mathrm{a} 50$ & \\
\hline & $\begin{array}{l}\text { Zone number or negative of material } \\
\text { number, } \\
\text { zone volume, } \\
\text { number of times unit is referenced in } \\
\text { the problem, } \\
\text { MatId }\end{array}$ & 2es14.6, 2i7 & $\begin{array}{l}\text { Note, if sensitivity data is } \\
\text { region-integrated, zone } \\
\text { number and zone volume are } \\
\text { both 0. If data is integrated } \\
\text { over all zones containing the } \\
\text { same material, the material } \\
\text { number is given in place of the } \\
\text { zone number as a negative } \\
\text { number. }\end{array}$ \\
\hline
\end{tabular}


Table 6.3.A.2. Format specification for TSUNAMI-B sensitivity data file (continued)

\begin{tabular}{|c|c|c|c|}
\hline Occurrence & Data & Format & Description \\
\hline & $\begin{array}{l}\text { energy integrated sensitivity } \\
\text { coefficient, } \\
\text { standard deviation for energy } \\
\text { integrated sensitivity coefficient, } \\
\text { sum of absolute value of group-wise } \\
\text { sensitivities, } \\
\text { sum of the group-wise } \\
\text { sensitivities with opposite sign as } \\
\text { energy integrated value (osc), } \\
\text { standard deviation for osc. }\end{array}$ & 5es14.6 & $\begin{array}{l}\text { Energy-integrated sensitivity } \\
\text { coefficients and their } \\
\text { standard deviations for this } \\
\text { profile. }\end{array}$ \\
\hline & group-wise sensitivity coefficients & $\begin{array}{l}\text { 5es14.6 (repeats } \\
\text { until all data is } \\
\text { printed) }\end{array}$ & $\begin{array}{l}\text { Energy-dependent sensitivity } \\
\text { coefficients. Begins with } \\
\text { highest energy group. }\end{array}$ \\
\hline & $\begin{array}{l}\text { standard deviation in group-wise } \\
\text { sensitivity coefficients }\end{array}$ & $\begin{array}{l}\text { 5es14.6 (repeats } \\
\text { until all data is } \\
\text { printed) }\end{array}$ & $\begin{array}{l}\text { Standard deviation for energy- } \\
\text { dependent } \\
\text { coefficients. Begins with } \\
\text { highest energy group. }\end{array}$ \\
\hline Once at end of file. & & & $\begin{array}{l}\text { Block of file verification } \\
\text { information. }\end{array}$ \\
\hline
\end{tabular}


$\begin{aligned} \text { sample } 3- & \text { tsunami-3d } \\ 238 & \text { number of neutron groups } \\ 3389 & \text { number of sensitivity profiles } \quad 291 \text { are region integrated }\end{aligned}$

$1.004674+/-0.000543 \mathrm{k}$-eff from the forward case energy boundaries:

2. $000000 \mathrm{E}+07$

$1.284000 \mathrm{E}+07$

$4.304000 \mathrm{E}+06$

$1.500000 \mathrm{E}+06$

$1.200000 \mathrm{E}+06$

$8.750000 \mathrm{E}+05$

$6.700000 \mathrm{E}+05$

$4.700000 \mathrm{E}+05$

$2.700000 \mathrm{E}+05$

$8.500000 \mathrm{E}+04$

$5.200000 \mathrm{E}+04$

$1.700000 \mathrm{E}+\odot 4$

3. $900000 \mathrm{E}+03$

2. $200000 \mathrm{E}+03$

$9.500000 \mathrm{E}+02$

$2.850000 \mathrm{E}+02$

1. $860000 \mathrm{E}+02$

1. $000000 \mathrm{E}+02$

7. $200000 \mathrm{E}+01$

$5.340000 \mathrm{E}+01$

4. $700000 \mathrm{E}+01$

3. $960000 \mathrm{E}+01$

3. $460000 \mathrm{E}+01$

3. $0000000 \mathrm{E}+01$

2. $000000 \mathrm{E}+01$

1. $509990 \mathrm{E}+01$

1. $150000 \mathrm{E}+01$

7. $000000 \mathrm{E}+00$

$5.400000 \mathrm{E}+00$

$3.500000 \mathrm{E}+00$

$2.870000 \mathrm{E}+\odot \odot$

$2.379990 \mathrm{E}+0 \odot$

$1.940000 \mathrm{E}+00$

$1.500000 \mathrm{E}+00$

$1.250000 \mathrm{E}+00$

$1.139990 \mathrm{E}+00$

1. $089990 \mathrm{E}+00$

1. $040000 \mathrm{E}+00$

9. $750000 \mathrm{E}-01$

8. $000000 \mathrm{E}-01$

6. $000000 \mathrm{E}-01$

3. $750000 \mathrm{E}-01$

2. $500000 \mathrm{E}-01$

1. $250000 \mathrm{E}-01$

6. $0000000 \mathrm{E}-02$
$1.733300 \mathrm{E}+07$

1. $000000 \mathrm{E}+07$

3. $000000 \mathrm{E}+06$

1. $400000 \mathrm{E}+06$

$1.100000 \mathrm{E}+06$

8. $611000 \mathrm{E}+05$

$6.000000 \mathrm{E}+05$

$4.400000 \mathrm{E}+05$

$2.000000 \mathrm{E}+05$

8. $200000 \mathrm{E}+04$

$5.000000 \mathrm{E}+04$

1. $300000 \mathrm{E}+04$

$3.740000 \mathrm{E}+03$

$1.800000 \mathrm{E}+03$

$6.830000 \mathrm{E}+02$

$2.400000 \mathrm{E}+02$

1. $220000 \mathrm{E}+02$

$9.000000 \mathrm{E}+01$

6. $750000 \mathrm{E}+01$

$5.200000 \mathrm{E}+01$

4. 520000E+01

3. $910000 \mathrm{E}+01$

$3.375000 \mathrm{E}+01$

$2.750000 \mathrm{E}+01$

1. $900000 \mathrm{E}+01$

1. $440000 \mathrm{E}+01$

1. $000000 \mathrm{E}+01$

$6.750000 \mathrm{E}+0 \odot$

$5.000000 \mathrm{E}+00$

$3.150000 \mathrm{E}+00$

$2.770000 \mathrm{E}+00$

2. $299990 \mathrm{E}+\odot \odot$

$1.860000 \mathrm{E}+0 \odot$

$1.450000 \mathrm{E}+0 \odot$

$1.224990 \mathrm{E}+\Theta \odot$

$1.129990 \mathrm{E}+0 \odot$

$1.080000 \mathrm{E}+00$

1. $030000 \mathrm{E}+0 \odot$

9. $500000 \mathrm{E}-01$

7. $5000000 \mathrm{E}-01$

5. $5000000 \mathrm{E}-01$

3. $500000 \mathrm{E}-01$

2. $250000 \mathrm{E}-01$

1. $0000000 \mathrm{E}-01$

5. $0000000 \mathrm{E}-\odot 2$
1. $568300 \mathrm{E}+07$

$8.187300 \mathrm{E}+06$

$2.479000 \mathrm{E}+06$

1. $356000 \mathrm{E}+06$

1. $010000 \mathrm{E}+06$

8. $200000 \mathrm{E}+05$

$5.730000 \mathrm{E}+05$

4. $200000 \mathrm{E}+05$

$1.500000 \mathrm{E}+05$

$7.500000 \mathrm{E}+04$

$4.500000 \mathrm{E}+04$

$9.5000000 \mathrm{E}+03$

$3.000000 \mathrm{E}+03$

$1.550000 \mathrm{E}+03$

$6.700000 \mathrm{E}+02$

$2.100000 \mathrm{E}+02$

1. $190000 \mathrm{E}+02$

8. $2000000 \mathrm{E}+01$

$6.500000 \mathrm{E}+01$

$5.060000 \mathrm{E}+01$

4. $400000 \mathrm{E}+01$

$3.800000 \mathrm{E}+01$

$3.325000 \mathrm{E}+01$

2. $500000 \mathrm{E}+01$

$1.850000 \mathrm{E}+01$

1. $375000 \mathrm{E}+01$

$9.099990 \mathrm{E}+00$

$6.500000 \mathrm{E}+00$

4. $750000 \mathrm{E}+00$

$3.049990 \mathrm{E}+0 \odot$

$2.669990 \mathrm{E}+0 \odot$

$2.209990 \mathrm{E}+0 \odot$

$1.770000 \mathrm{E}+0 \odot$

$1.400000 \mathrm{E}+\odot \odot$

$1.200000 \mathrm{E}+0 \odot$

$1.120000 \mathrm{E}+00$

$1.070000 \mathrm{E}+00$

1. $020000 \mathrm{E}+00$

9. 250000E- 01

7. $000000 \mathrm{E}-01$

5. $0000000 \mathrm{E}-01$

3. $2500000 \mathrm{E}-01$

2. $0000000 \mathrm{E}-01$

9. $0000000 \mathrm{E}-02$

4. $0000000 \mathrm{E}-02$
1. $455000 \mathrm{E}+07$

$6.434000 \mathrm{E}+06$

$2.354000 \mathrm{E}+06$

1. $317000 \mathrm{E}+06$

$9.200000 \mathrm{E}+05$

$7.500000 \mathrm{E}+05$

$5.500000 \mathrm{E}+05$

4. $0000000 \mathrm{E}+05$

1. $283000 \mathrm{E}+05$

$7.300000 \mathrm{E}+04$

$3.000000 \mathrm{E}+04$

$8.030000 \mathrm{E}+03$

$2.580000 \mathrm{E}+03$

$1.500000 \mathrm{E}+03$

$5.500000 \mathrm{E}+02$

$2.075000 \mathrm{E}+02$

1. $150000 \mathrm{E}+02$

8. $000000 \mathrm{E}+01$

$6.100000 \mathrm{E}+01$

$4.920000 \mathrm{E}+01$

4. $240000 \mathrm{E}+01$

$3.700000 \mathrm{E}+01$

$3.175000 \mathrm{E}+01$

$2.250000 \mathrm{E}+01$

$1.700000 \mathrm{E}+01$

1. $290000 \mathrm{E}+01$

$8.099990 \mathrm{E}+0 \odot$

$6.250000 \mathrm{E}+00$

$4.000000 \mathrm{E}+00$

$3.000000 \mathrm{E}+00$

$2.570000 \mathrm{E}+0 \odot$

$2.120000 \mathrm{E}+00$

$1.679990 \mathrm{E}+\odot \odot$

$1.349990 \mathrm{E}+\odot \odot$

$1.174990 \mathrm{E}+\odot \odot$

$1.110000 \mathrm{E}+00$

$1.059990 \mathrm{E}+0 \odot$

1. $009990 \mathrm{E}+00$

9. $0000000 \mathrm{E}-01$

$6.500000 \mathrm{E}-01$

4. $5000000 \mathrm{E}-01$

3. $000000 \mathrm{E}-01$

1. 750000E- 01

8. $000000 \mathrm{E}-02$

3. $000000 \mathrm{E}-02$
1. $384000 \mathrm{E}+07$

$4.800000 \mathrm{E}+06$

$1.850000 \mathrm{E}+06$

$1.250000 \mathrm{E}+06$

$9.000000 \mathrm{E}+05$

$6.790000 \mathrm{E}+05$

$4.995200 \mathrm{E}+05$

3. 30000०E+05

1. $0000000 \mathrm{E}+05$

$6.000000 \mathrm{E}+04$

$2.5000000 \mathrm{E}+04$

$6.000000 \mathrm{E}+03$

2. $290000 \mathrm{E}+03$

$1.150000 \mathrm{E}+03$

3. $050000 \mathrm{E}+02$

1. $925000 \mathrm{E}+02$

$1.080000 \mathrm{E}+02$

7. $6000000 \mathrm{E}+01$

$5.900000 \mathrm{E}+01$

4. $8300000 \mathrm{E}+01$

$4.100000 \mathrm{E}+01$

$3.550000 \mathrm{E}+01$

$3.125000 \mathrm{E}+01$

2. $100000 \mathrm{E}+01$

1. $600000 \mathrm{E}+01$

$1.190000 \mathrm{E}+01$

$7.150000 \mathrm{E}+00$

$6.000000 \mathrm{E}+00$

$3.730000 \mathrm{E}+00$

$2.969990 \mathrm{E}+0 \odot$

2. $469990 \mathrm{E}+0 \odot$

2. $000000 \mathrm{E}+0 \odot$

1. $589990 \mathrm{E}+0 \odot$

$1.299990 \mathrm{E}+\odot \odot$

1. $150000 \mathrm{E}+0 \odot$

1. $099990 \mathrm{E}+00$

1. $049990 \mathrm{E}+0 \odot$

1. $000000 \mathrm{E}+\odot \odot$

8. 500000E- 01

6. $250000 \mathrm{E}-01$

4. $000000 \mathrm{E}-01$

$2.750000 \mathrm{E}-01$

1. $500000 \mathrm{E}-01$

7. $0000000 \mathrm{E}-02$

2. 530000E- 02

Figure 6.3.A.2. Truncated sensitivity data file for LEU-COMP-THERM-009 sample problem. 


\begin{tabular}{|c|c|c|c|c|}
\hline 1. $000000 \mathrm{E}-02$ & $0 \odot \odot \odot \odot E-\odot 3$ & $5.000000 \mathrm{E}-\odot 3$ & 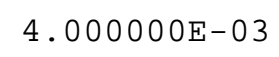 & 0.000 \\
\hline $2.500000 \mathrm{E}-\odot 3$ & 2. $.0 \odot \odot \odot \odot E-\odot 3$ & 1. $5000 \odot \odot E-\odot 3$ & $12 \rho ค \ominus \odot \odot F-03$ & \\
\hline$\odot \odot \odot \odot \odot E-\odot 4$ & $5.0 \odot \odot \odot \odot \odot E-\odot 4$ & 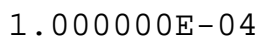 & 1. $00 \odot \odot \odot \odot E$ & \\
\hline & & & & \\
\hline$\odot$ & & & & \\
\hline$\odot .00 \odot \odot$ & $\odot . \odot \odot \odot \odot \odot \odot E+\odot \odot$ & $\odot$ & & \\
\hline $5.878199 \mathrm{E}-01$ & 1. $\odot 80812 \mathrm{E}-\odot 2$ & $93673 E-01$ & -2.577 & $6.213759 \mathrm{E}-0$ \\
\hline$\odot . \odot \odot \odot \odot \odot \odot E+\odot \odot$ & $5.495367 \mathrm{E}-07$ & $2.331587 \mathrm{E}-06$ & $3.300773 \mathrm{E}-\odot 6$ & 1. $013678 \mathrm{E}-05$ \\
\hline $51 E-\odot 4$ & $8 E-\odot 4$ & $179 E-\odot 3$ & $19 E-\odot 2$ & \\
\hline $3059 E-02$ & $4 \mathrm{E}-02$ & $.750866 E-\odot 3$ & $85 E-02$ & \\
\hline $5 E-\odot 3$ & $2 E-03$ & $62922 E-\odot 3$ & $9 E-\odot 3$ & -03 \\
\hline E- -03 & $E-\odot 3$ & $93 E-03$ & ०० - ๑3 & \\
\hline $1.846754 \mathrm{E}-\odot 3$ & $5.699152 \mathrm{E}-\odot 3$ & $1.105599 \mathrm{E}-02$ & 1. 187824E- $\odot 2$ & $1.369476 \mathrm{E}-03$ \\
\hline 1. 163906E-๑2 & อ338E- - 03 & 4. $09 \odot 5 \odot 4 E-\odot 3$ & $14 E-03$ & $6 E-03$ \\
\hline$E-03$ & -03 & $22 E-03$ & & \\
\hline$E-\odot 2$ & -02 & 77E- 03 & $9 E-03$ & \\
\hline 1.1 & -03 & $2 E-\odot 4$ & $8 E-03$ & \\
\hline$E-03$ & $E-03$ & $7 E-03$ & $8 E-\odot 3$ & $=-03$ \\
\hline E- - 03 & $2 \mathrm{E}-03$ & $83 E-03$ & $21 E-03$ & $9 E-03$ \\
\hline$-\odot 4$ & $=-03$ & OE - 03 & $8 E-03$ & \\
\hline 3. & 3 & $E-\odot 4$ & 3 & \\
\hline & -04 & $7 E-03$ & $E-\odot 3$ & \\
\hline 2.2 & & $E-\odot 4$ & $E-\odot 4$ & \\
\hline 7.128 & 4.2 & $98 E-04$ & $4 E-\odot 3$ & -03 \\
\hline $3 E-03$ & -04 & $4 E-04$ & $\odot E-\odot 4$ & E- $\odot 4$ \\
\hline-03 & 3. & $E-\odot 4$ & $E-\odot 4$ & \\
\hline 1. & 4 & $=-\odot 4$ & $=-04$ & \\
\hline & & & & \\
\hline$E-\odot 4$ & 6. & $8 E-04$ & $E-\odot 4$ & -04 \\
\hline 3.6204 & 2.2 & $22 E-\odot 4$ & $1 E-\odot 4$ & פE- -04 \\
\hline & & $=-03$ & & \\
\hline 8. & 4 & 3 & $E-04$ & \\
\hline 1. & 4. & E- & $E-03$ & 04 \\
\hline$\odot 4$ & $-\odot 3$ & $7 E-\odot 3$ & $E-\odot 3$ & $E-\odot 4$ \\
\hline$E-\odot 4$ & $E-04$ & OE - ๑4 & $8 E-\odot 4$ & $2 E-03$ \\
\hline & & $8 E-03$ & $\mathrm{E}-03$ & $6 E-04$ \\
\hline 1.6 & 4. & 4 & 4 & \\
\hline & & 4 & & \\
\hline & & $3 E-$ & 8 & \\
\hline$E-\odot 4$ & $E-\odot 4$ & 97E- - 4 & $3 E-\odot 4$ & $7 E-03$ \\
\hline E- -4 & $E-\odot 4$ & $2 E-\odot 4$ & $1 E-\odot 4$ & $E-\odot 3$ \\
\hline & & $E-\odot 4$ & & $E-04$ \\
\hline 7.1 & & ЭE- - 3 & & $E-\odot 3$ \\
\hline & & & & \\
\hline & & 5 & 3 & $-\mathrm{C}^{-1}$ \\
\hline$E-\odot 3$ & $E-03$ & $5 E-\odot 4$ & $5 E-\odot 3$ & $6 E-03$ \\
\hline 1.0 & E- - & $521 E-04$ & $72 E-\odot 4$ & $5.043924 E-04$ \\
\hline & & $73 E-04$ & $7 \mathrm{E}$ & OL- \\
\hline 4.5 & & उ०E- - 44 & $5 \mathrm{E}-$ & \\
\hline 1. 292846E- $\odot 3$ & $3630 E-03$ & . 487261E- 03 & 1. 447523E- $\odot 3$ & $2.061059 \mathrm{E}-\odot$ \\
\hline
\end{tabular}

Figure 6.3.A.2. Truncated sensitivity data file for LEU-COMP-THERM-009 sample problem. (continued) 


\begin{tabular}{|c|c|c|c|c|}
\hline 93 & 4 & 4 & 3E-05 & \\
\hline & . . 516055E- - & $-4.246086 E-03$ & $-2.081466 \mathrm{E}-03$ & $-9.899241 E-$ \\
\hline$E-\odot 3$ & $-1.532964 E-03$ & $959768 E-\odot 4$ & 4 & \\
\hline 04 & -1.851 & & 5 & \\
\hline 05 & -6.599 & & & \\
\hline$E+\odot \odot$ & & & 7 & \\
\hline 6 & & & & \\
\hline (1) & 6.9590 & $2.559563 E-\odot 4$ & & (2) \\
\hline 4 & & & & \\
\hline 4 & 4. & & & \\
\hline & & & & \\
\hline & & & & \\
\hline-04 & $E-\odot 4$ & $E-\odot 4$ & & \\
\hline & & & & \\
\hline$E-\odot 4$ & & & & \\
\hline & & & & \\
\hline & 1.5 & & & \\
\hline & & & & \\
\hline & 8.1 & & & \\
\hline & & & & \\
\hline 4 & & & & \\
\hline 3 & 1. & 2 . & & \\
\hline 04 & 4. & & 4 & \\
\hline & & & & \\
\hline & & & & \\
\hline & & & & 2 \\
\hline 4 & 1 & & & \\
\hline & & & & \\
\hline & & & & \\
\hline & & & & \\
\hline & & & & \\
\hline & & & & \\
\hline & & & & \\
\hline 4 & 2. & 4 & & \\
\hline & & & & \\
\hline & & & & \\
\hline & & & & \\
\hline & & & & \\
\hline & & & & \\
\hline & & & & \\
\hline & & & & \\
\hline & & & & \\
\hline 5 & 4.5 & $5 E-05$ & -05 & 1. \\
\hline & & & & \\
\hline & & & & \\
\hline & & & & \\
\hline & & & & \\
\hline & & 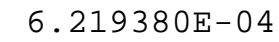 & & \\
\hline $10405-33$ & 1. $.881433 E-03$ & 1. 278081E- - & $1.529288 \mathrm{E}-0$ & \\
\hline
\end{tabular}

Figure 6.3.A.2. Truncated sensitivity data file for LEU-COMP-THERM-009 sample problem. (continued) 


\begin{tabular}{|c|c|c|c|c|}
\hline $2.160035 \mathrm{E}-03$ & $173668 \mathrm{E}-03$ & $2.749914 \mathrm{E}-03$ & $1.747805 \mathrm{E}-03$ & $2.894034 \mathrm{E}-03$ \\
\hline $7.595401 \mathrm{E}-\odot 4$ & $.846859 E-\odot 4$ & $2.217471 \mathrm{E}-\odot 4$ & 1. $914071 \mathrm{E}-\odot 4$ & $9.697134 \mathrm{E}-05$ \\
\hline $8.595824 \mathrm{E}-05$ & $688035 E-05$ & $872943 E-05$ & 05 & \\
\hline $222 E-05$ & $3262 E-05$ & 777834E & & \\
\hline \multirow[t]{2}{*}{$\mathrm{h}-1$} & scatter & 100 & & \\
\hline & & & & \\
\hline$E+\odot \odot$ & ๑०९०९E+๑९ & 0 & & \\
\hline $6.303991 \mathrm{E}-01$ & 1. $077414 \mathrm{E}-02$ & $4 \odot \odot 794 E-\odot 1$ & $-4.840258 E-\odot 3$ & $4.481088 \mathrm{E}-$ \\
\hline$E+\odot \odot$ & $1 E-07$ & $331707 E-\odot 6$ & & \\
\hline $4523 E-\odot 4$ & $4 E-\odot 4$ & . $936591 E-\odot 3$ & $89 E-02$ & \\
\hline E- - 2 & $3 E-02$ & 751186E- - 3 & $26 E-02$ & \\
\hline & $9 E-\odot 3$ & $91 E-03$ & & \\
\hline $9.353111 \mathrm{E}-\odot 3$ & $6.456018 \mathrm{E}-\odot 3$ & $6.330305 \mathrm{E}-03$ & $2.219428 \mathrm{E}-03$ & 47952E- - \\
\hline $3 E-\odot 3$ & $83 E-03$ & $62 E-02$ & $883 E-\odot 2$ & $54 E-03$ \\
\hline E- $\odot 2$ & $E-03$ & $26 \mathrm{E}-03$ & & \\
\hline$E-\odot 3$ & $E-\odot 3$ & $4 E-\odot 3$ & $\odot 2$ & \\
\hline$E-\odot 2$ & $E-02$ & $3 E-03$ & 93 & \\
\hline$E-\odot 3$ & $3 E-03$ & $30 E-04$ & $E-03$ & $E-\odot 3$ \\
\hline $4 E-03$ & $57 E-03$ & $30 E-03$ & $25 E-03$ & $7 E-03$ \\
\hline$=-03$ & $E-\odot 3$ & $3 E-03$ & & \\
\hline$-\odot 4$ & -03 & $5 E-\odot 3$ & & \\
\hline & $E-03$ & $8 E-\odot 4$ & & \\
\hline$E-\odot 3$ & & 03 & & \\
\hline LE- -3 & $1 E-03$ & $28 \mathrm{E}-04$ & $E-\odot 4$ & \\
\hline$E-03$ & $\mathrm{DE}-04$ & $78 E-04$ & $9 E-03$ & $E-03$ \\
\hline-03 & -04 & $3 E-04$ & & \\
\hline-03 & -04 & $=-\odot 4$ & & \\
\hline & & & & \\
\hline & $E-03$ & $7 \mathrm{E}-\odot 4$ & 93 & $-\odot 4$ \\
\hline$E-\odot 4$ & $E-\odot 4$ & 71E- - 4 & $=-\odot 4$ & $E-\odot 4$ \\
\hline$-\odot 4$ & $E-04$ & $E-\odot 4$ & & \\
\hline-03 & 1. & E-०3 & & \\
\hline$=-04$ & $E-\odot 4$ & $8 E-\odot 3$ & $E-\odot 4$ & $E-\odot 4$ \\
\hline-03 & -04 & $E-\odot 3$ & $E-\odot 3$ & $E-\odot 4$ \\
\hline$E-\odot 4$ & $E-\odot 3$ & $38 E-03$ & $94 E-03$ & $27 E-04$ \\
\hline & & $9 E-\odot 4$ & & $\odot E-\odot 3$ \\
\hline 1.2 & $E-\odot 4$ & $3 E-\odot 3$ & 3 & \\
\hline & & $=-\odot 4$ & & \\
\hline & 4 & 4 & & \\
\hline$E-\odot 4$ & $96 E-\odot 4$ & $83 E-\odot 4$ & $4 E-\odot 4$ & คE - -44 \\
\hline$E-\odot 4$ & $9 E-04$ & $1 E-\odot 4$ & $9 E-\odot 3$ & $5 E-03$ \\
\hline 7 & $E-04$ & $E-\odot 4$ & & $E-\odot 3$ \\
\hline & & & & \\
\hline & & & & \\
\hline & & $-\odot 3$ & & \\
\hline$E-\odot 3$ & $5 E-03$ & $\odot E-\odot 3$ & $7 \mathrm{E}-$ & $3 E-03$ \\
\hline $2 E-03$ & $07 E-\odot 3$ & $9.137678 \mathrm{E}-04$ & $992 \mathrm{E}-03$ & $6 E-03$ \\
\hline 3 & $=-03$ & $4 \mathrm{E}-03$ & 6.2 & +ᄃ \\
\hline-03 & $E-03$ & $6 E-03$ & $29 E-03$ & 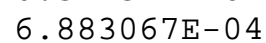 \\
\hline $777 E-\odot 4$ & . 486881E-๑4 & $9.267605 \mathrm{E}-\odot 4$ & 1. $016119 E-03$ & $1.337179 E-\odot$ \\
\hline
\end{tabular}

Figure 6.3.A.2. Truncated sensitivity data file for LEU-COMP-THERM-009 sample problem. (continued) 


\begin{tabular}{|c|c|c|c|c|}
\hline$E-\odot 3$ & $7 E-\odot 3$ & $19 E-\odot 3$ & $269599 E-\odot 3$ & \\
\hline $6744 E-03$ & $51763 E-03$ & 488E- 03 & & \\
\hline 93 & $24868 E-03$ & $7 \mathrm{E}$ & $61996 \mathrm{E}$ & $0 \Omega$ \\
\hline & & & & \\
\hline & 95 & & 5 & \\
\hline & & & & \\
\hline & & & & \\
\hline$E-06$ & $3 E-05$ & & & \\
\hline & $6.958902 \mathrm{E}$ & 4 & $31330 E-\odot 4$ & $389 \mathrm{E}$ \\
\hline & & & & \\
\hline 4 & 4 & & & \\
\hline & & & & \\
\hline & & & & \\
\hline$-\odot 4$ & $8 E-\odot 4$ & & & $6 \mathrm{E}$ \\
\hline-03 & $E-03$ & & & \\
\hline$-\odot 4$ & & & & \\
\hline & & & & \\
\hline & & & & \\
\hline & & & & \\
\hline & & & & \\
\hline & & & & \\
\hline 4 & 7 & & & 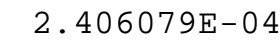 \\
\hline 3 & & & & \\
\hline & & & & \\
\hline & & & & \\
\hline & & & & \\
\hline & & & & \\
\hline & & & & \\
\hline 4 & & & & \\
\hline & & & & \\
\hline & & & & 3 \\
\hline & & & & \\
\hline & & & & \\
\hline & & & & \\
\hline & & & & 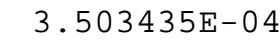 \\
\hline & & & & \\
\hline 2.2 & & 04 & 04 & \\
\hline & & & & \\
\hline & & & & \\
\hline & & & & \\
\hline & & & & \\
\hline & & & & \\
\hline$=-05$ & LE - $\odot 5$ & E- 05 & E- 05 & 2. \\
\hline-05 & $9 \mathrm{E}-05$ & E- 05 & E- 05 & $v<L$ \\
\hline & & & & \\
\hline & & & & \\
\hline & & & & \\
\hline & & & & \\
\hline & प्रॉ- - 4 & $0.10000<[-04$ & $0.109344[-04$ & \\
\hline
\end{tabular}

Figure 6.3.A.2. Truncated sensitivity data file for LEU-COMP-THERM-009 sample problem. (continued) 


\begin{tabular}{|c|c|c|c|c|}
\hline $1.577516 \mathrm{E}-03$ & $1.075698 \mathrm{E}-03$ & $1.270736 \mathrm{E}-03$ & $1.520277 \mathrm{E}-03$ & $79 E-03$ \\
\hline 2.1 & $7027 E-03$ & $2.729725 \mathrm{E}-\odot 3$ & $1.737714 \mathrm{E}-03$ & $629 E-03$ \\
\hline & $E-\odot 4$ & & & 05 \\
\hline & $E-05$ & $4.814730 \mathrm{E}-05$ & $918 E-05$ & 3.4 \\
\hline 1 & $3.212018 \mathrm{E}-05$ & $8.655023 \mathrm{E}-$ & & \\
\hline
\end{tabular}

$\ldots$

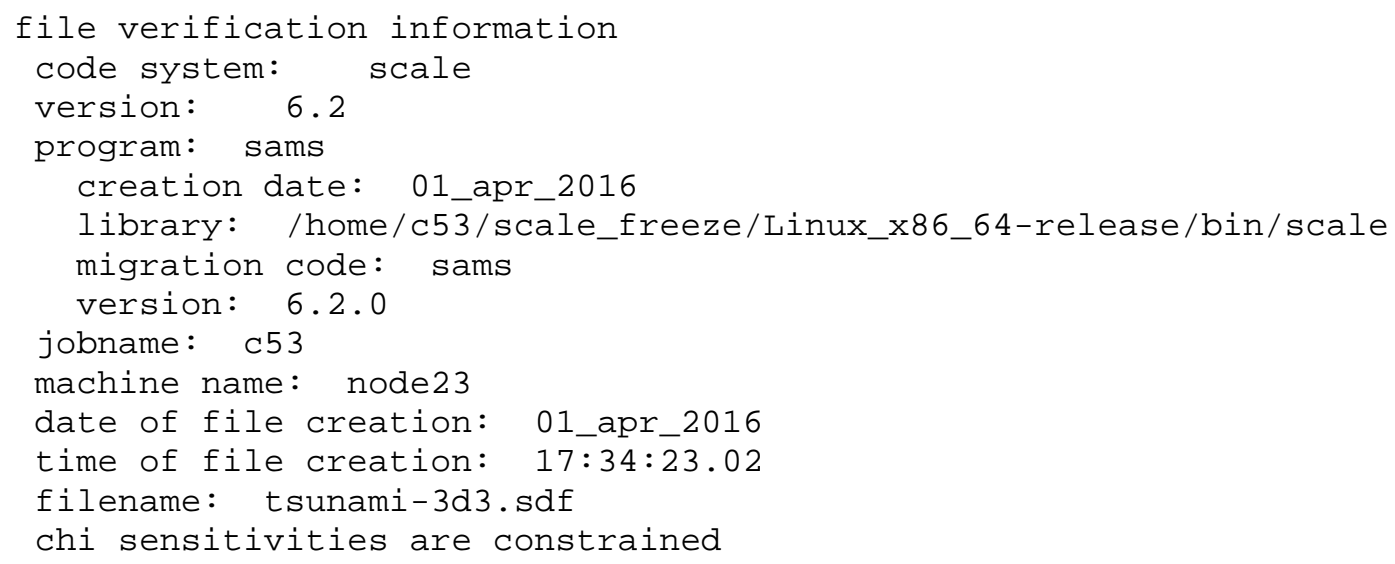

Figure 6.3.A.2. Truncated sensitivity data file for LEU-COMP-THERM-009 sample problem. (continued) 